\title{
ANÁLISIS DE LA PREVALENCIA DE MB2 EN PRIMEROS MOLARES SUPERIORES PERMANENTES MEDIANTE EL USO DEL ESTEREOMICROSCOPIO, LA INSPECCIÓN VISUAL Y VALORACIÓN TOPOGRÁFICA DEL PISO DE CÁMARA PULPAR - IN VITRO.
}

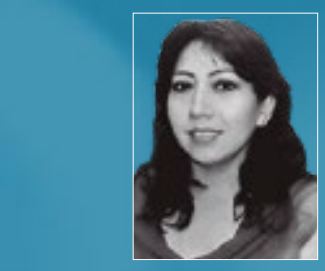

Dra. Erika Espinosa Directora - Postarado Endodoncia Universidad Central Del Ecuador

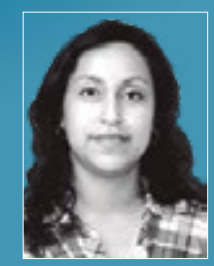

Odont. Jéssica Llano Posgrado de Endodoncia. Facultad de Od Fultad de Odontología Universidad Central del Ecuador

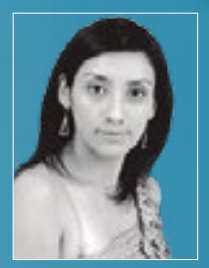

Odont. Mónica Pavón Posgrado de Endodoncia. Facultad de Odontología Universidad Central del Ecuador

\section{INTRODUCCIóN}

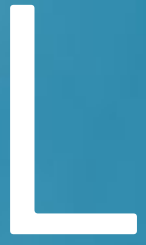

La raíz mesiobucal y la posibilidad de un segundo conducto en los primeros molares superiores ha despertado el interés de muchos odontólogos especialistas (1). Es importante conocer la anatomía normal y sus diferentes variaciones para estar en la capacidad de reconocer clínicamente las mismas, ya que un conducto no identificado es imposible limpiar llevándonos al fracaso en la práctica Endodóntica. (2)

La evidencia clínica ha demostrado que la magnificación a través del uso del microscopio facilita la ubicación del orificio de entrada de los canales radiculares incluido el MB2. Según la literatura la identificación de un cuarto canal en los molares superiores a simple vista es mucho más compleja, representando el 20 al 38\% MB2 identificado a través del microscopio dental es más alta, oscilando entre el 50.4\% hasta 95\% (3).

La distancia existente entre el orificio MB1 y MB2 ha sido considerada otra referencia a ser tomada en cuenta durante la determinación de un cuarto conducto en el primer molar superior permanente, la literatura manifiesta una variación de dicha distancia entre 1 mm a 5 mm. (1-5).

Topográficamente a nivel del piso de la cámara pulpar se ha observado la presencia de unas depresiones o surcos con diferentes formas llamadas rostrum canalium, importantes para la localización del MB2. La literatura refiere la disposición de los surcos a nivel del piso en forma de: a.depresiones en forma de $Y$ con entrada a tres conductos b. surcos uniendo el orificio MB con DB, c.- orificios individuales unidos por un surco, d.- otras formas con menor frecuencia. (4-9) FIG. 4
Este estudio tiene la finalidad de identificar la prevalencia de MB2 mediante varios métodos para facilitar la localización clínica de este cuarto conducto al momento de realizar un tratamiento de Endodoncia.

El objetivo de este estudio fue valorar la presencia y prevalencia del conducto MB2 en primer molar superior a través de un estereomicroscopio la medición de la distancia que existe entre MB1 - MB2 y la valoración de las diferentes variaciones en la forma del rostrum canalium en piso de cámara pulpar.

\section{MATERIALES Y MÉTODOS}

Cuarenta primeros molares superiores permanentes humanos extraídos recogidos aleatoriamente fueron seleccionados para este estudio. Para estandarizar la muestra fueron cortados por debajo de la unión cemento esmalte utilizando un disco de diamante. Se retiró cálculos, tejido óseo con una cuchareta (Destply- Maillerfer), luego se realizó la desinfección de los especímenes sumergiéndolos en hipoclorito de sodio al 5,25\% durante 24 horas para remover el tejido orgánico, posteriormente los dientes se lavaron abundantemente con agua para ser manipulados.

Las muestras fueron examinadas indistintamente por dos operadores para observar a simple vista la presencia del MB2 procedimiento que fue realizado utilizando un explorador Dg-16 de punta fina (Destply- Maillerfer) y una lima K-FILE \#6 a \#10 (Destply- Maillerfer) respectivamente dependiendo del tamaño del orificio. 


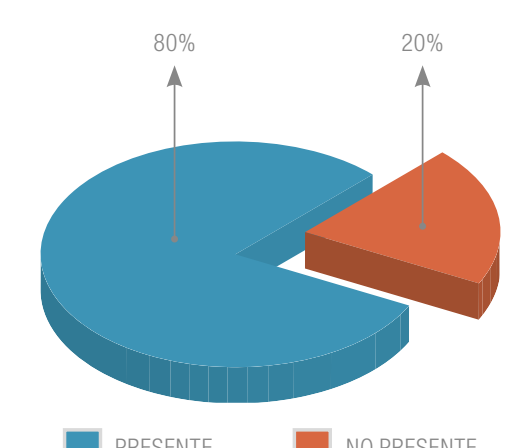

PRESENTE NO PRESENTE

FlG 1. Prevalencia del MB2 en primeros
molares superiores permanentes.

Los especímenes fueron fijados sobre un bloque de plastilina y llevados al lente del estéreomicroscopio marde Patología de la Facultad de Odontología de la Universidad Central de Ecuador con una magnificación de $2.5 \mathrm{X}$.

Una vez encontrado todos los orificios de entrada a los conductos se valoró la distancia existente entre el MB1 yB2 utilizando un compás doble punta y se trasladó la medida a una regla milimétrica para su evaluación.

Para observar el rostrum canalium se realizo la inspección directa de la muestra valorando el cambio de color del piso de camara pulpar y la forma de los surcos respecto a los orificios de entrada de los conductos.

\section{RESULTADOS}

o bucales anaizadas se encontraron 32 que tenían un cuarto canal correspondiente a 80\%, y un 20\% representado por 8 dientes en los que no estuvo presente el canal MB2. Fig. 1. Sin embargo solo 13 dientes que son el $32 \%$ fueron detectados a simple vista y 27 dientes fueron observados con la ayuda del estereomicroscopio que fue el $68 \%$ Fig. 2 y 3

De las 40 muestras en las que se analizo el piso de camara pulpar se pudo observar que el rostrum cana-

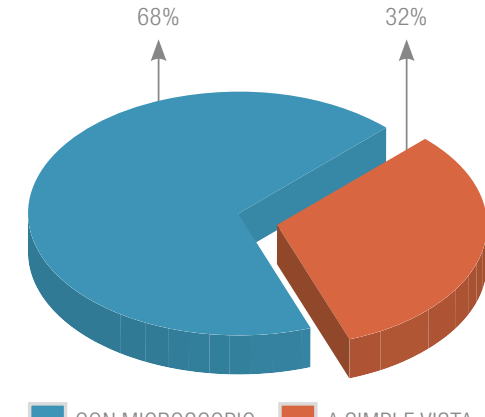

CON MICROSCOPIO A A SIMPLEVISTA

FIG 2. Incidencia en la detección de MB2
mediante esteriomicroscosoio y a simple vista.

Fig 3. Conductos existentes en un primer
molar superior permanente.

\begin{tabular}{|c|c|c|}
\hline \multicolumn{3}{|c|}{$\begin{array}{l}\text { DISPOSICIÓN DEL } \\
\text { ROSTRUM CANALIUM }\end{array}$} \\
\hline Disposición & \#\# dientes & $\%$ \\
\hline $\begin{array}{l}\text { A. depresiones } \\
\text { en forma de } Y\end{array}$ & 4 & $10 \%$ \\
\hline $\begin{array}{l}\text { B. Forma de Y } \\
\text { unido MB -DB }\end{array}$ & 2 & $5 \%$ \\
\hline $\begin{array}{l}\text { C. orificios individuales } \\
\text { unidos por un surco }\end{array}$ & 4 & $10 \%$ \\
\hline $\begin{array}{l}\text { B. Forma de } Y \\
\text { unido } M B-D B\end{array}$ & 31 & $78 \%$ \\
\hline D. Otras formas & 3 & $8 \%$ \\
\hline TOTAL & 40 & $100 \%$ \\
\hline
\end{tabular}

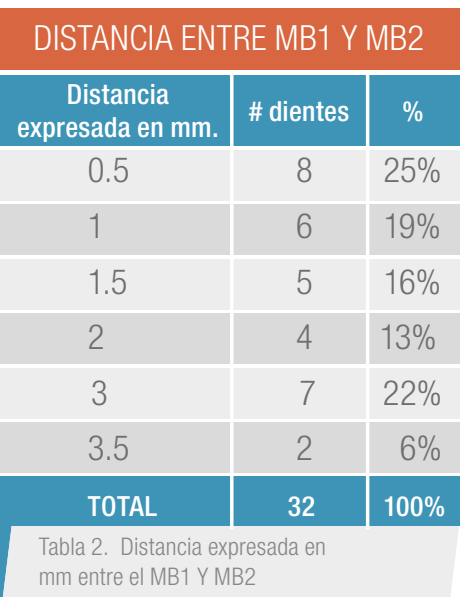

liun tuvo las siguientes disposiciones 4 dientes con depresiones en forma de $Y(10 \%), 2$ dientes en los que orificio MB se une con el DB $(5 \%$. 31 dientes en los cuales existen orifcios individuales unidos por un surco siendo la prevalencia más alta con un $78 \%$ y encontrándose 3 dientes co otras formas variadas poco frecuentes (8\%). Tabla 1. Fig. 4 y 5

Al realizar la medición en milíme tros de la distancia existente entre MB1 y MB2 de los especimenes que presentaron un cuarto canal, los sultados fueron. 0,5 mIm midieron 5 dientes que representan el $25 \%$ de la muestra siendo esta la prevalencia más alta, 1 mmm correspondió a 6 dientes $(19 \%), 1,5 \mathrm{~mm} 5$ dientes $(16 \%), 2 \mathrm{~mm} 4$ dientes $(13 \%), 3 \mathrm{~mm}$ 7 dientes (22\%) y 3,5 mm 2 dientes (6\%). Tabla 2. Figura 6

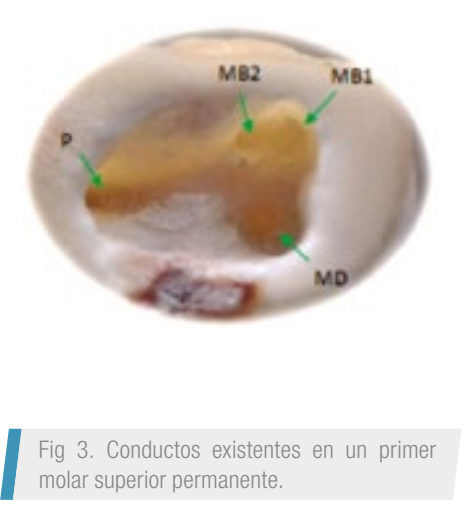

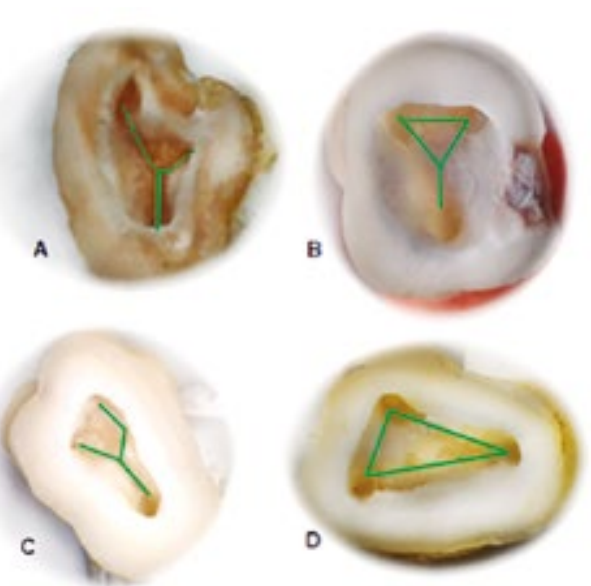

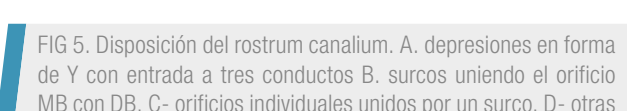

de $Y$ con entrada a tres conductos $B$, surcos uniendo el orfiticio
$M B$ con $D B, C$ - onfícicos individuales unidos por un surco, D- otras
tormas con menor frecuencia.

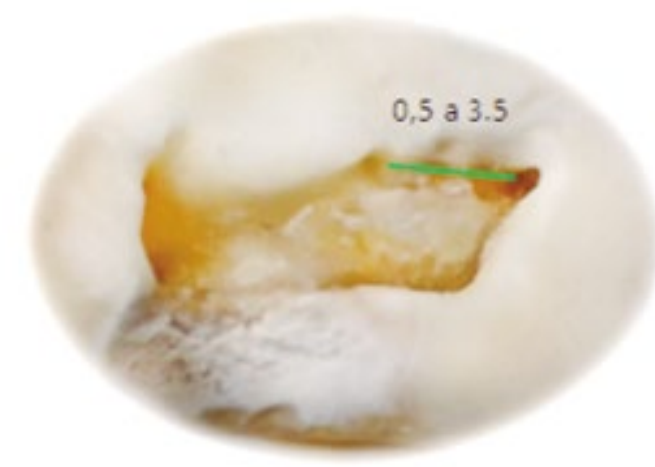

Fig. 6. Distancia entre mb1 y mb2

\section{DISCUSIÓN}

El conducto MB2 fue elegido para cidencia y sin embargo en much ocasiones suele ser dificil encontrarlo. (1)

La literatura refiere que la morfologia de raíz mesiobucal de los molares superiores presenta un sistema radicular complejo lo que hace que se pre sente un segundo canal como una de las variaciones mas comunes. (7)

Varios estudios realizados han demostrado la incidencia de un segundo canal en la raiz mesiobucal de los primeros molares superiores en un $18.6 \%$ a $96.1 \%$ dependiendo de los métodos utilizados, visión directa o con microscopio respectivamente. (2). Diferentes estudios indican que el número de MB2 de los ros molares superiores ha cambiad respecto de estudios recientes donde indica que su presencia es muy alta. Kulild y Peters indican que el primer molar superior tiene un alto indice de fracaso en los dientes posteriores 10 cual se atribuye a la prevalencia de un cuarto conducto. $(8$

En el $80 \%$ de los primeros molares superiores incluidos en este estudio se observó el orificio de entrada al conducto MB2, confirmando con esta Unormación la prevalencia elevada de

Según las referencias bibliográficas el MB2 puede encontrarse con mayor $(60.5 \%)$ que en un estuct $(18-36 \%)$. (2-7-10)

La importancia del uso del microscopio para la detección de los orificios del conducto mesiobucal en dientes extraidos en comparación con el anailisis a simple vista indica que el microscopio incrementa la posibilidad de encontrar MB2 del 51- 82\%. Ademas se debe recalcar que al hacer un estudio clinico con microscopio, lupas y a simple vista el microscopio sigue teniendo mayor eficacia en la localización del MB2. (11-12-13. En nuestro estudio el uso del estereomicroscopio en relacion al analisis a simple vista ha tenido una incidencia del $68 \%$ y $32 \%$ sin la utilización

El orificio de entrada al conducto MB2 se dispone entre el MB1 y el conducto palatino. La distancia existente en los conductos MB1 y MB2 puede variar entre $0,3 \mathrm{~mm}$ y $3,8 \mathrm{~mm}$ siendo muy próximos estos dos orificios pudiendo haber un fallo en I detección del MB2. Otros autores determinan que la distancia media entre os orificios MB1 y MB2 fue de 1 a 5

En este estudio la distancia prome dio entre MB1 y MB2 de las muestra analizadas fue de $0,5 \mathrm{~mm}$ a $3 \mathrm{~mm}, \mathrm{si}-$ milar a lo reportado por otros autores.

Se ha observado una intima relación entre los surcos del piso de la cámara y la entrada a los conductos radiculares, esta relación indica que operador debe tener la precaucion de explorar la anatomia del piso para tacilitar la localizacion de los orificios de entrada a los conductos radiculares durante el tratamiento de Endodoncia. Además la literatura menciona la presencia de formación de dentina secundaria en la raiz mesio bucal a nivel del piso lo cual obstruye la entra a los conductos. (1-4)

La revisión bibliográfica señala que la disposición que presentan los canales del piso de la cámara pulpar son un elemento importante por to tanto no se debe pasar por alto su valora- 
ción durante la localización del conducto MB2. Conocer la anatomía del piso de cámara pulpar facilitará la identificación con exactitud del MB2. (9). La exploración de los surcos del piso de la cámara mediante un explorador endodóntico puede llevarnos a localizar orificios secundarios. (1)

Acosta describe las diferentes formas que se marcan en el fondo del piso de cámara pulpar que son consideradas en la localización del MB2, así como del resto de conductos. Confirmando lo descrito anteriormente, la presencia de estos surcos con ciertas disposiciones que suelen ser constantes y que en pocas ocasiones adoptan formas geometrías, las muestras analizadas en este estudio nos dan una prevalencia de: a.- depresiones en forma de $Y$ con entrada a tres conductos (10\%) b. surcos uniendo el orificio MB con DB (5\%), c.- orificios individuales unidos por un surco (78\%) y d.- otras formas (8\%).

\section{CONCLUSIÓN}

La prevalencia de mb2 en primeros molares superiores es elevando por lo que obligación del especialista es buscar ya sea por referencias anatómicas o mediante el uso de recursos tecnológicos la presencia del mismo.

\section{REFERENCIA}

1. Görduysus M, Görduysus M, Friedman S. Operating microscope improves negotiation of second mesiobuccal canals in maxillary molars. Journal of Endodontics 2001; Vol. 27, No. 11, $683-686$

2. Raiput F, Kalhoro F, Shaikh M, Khatoon S. Validity of different methods for mb-2 canal location in permanent maxillary molars Pakistan Oral \& Dental Journal 2014; Vol 34, No. 3, 548-551.

3. Pais A, et al. Sodium fluorescein and cobalt blue filter coupled to a dental operating microscope to optimise root canal location in maxillary first molars. ENDO (Lond Engl) 2014; 8(3):193-198.

4. Acosta $\mathrm{S}$, Bosaans T. Anatomy of the pulp chamber. Journal of Endodontics. 1978; VOL 4, 215-219

5. Ting P. C. S, NGA. L. Clinical detection of the minor mesiobuccal canal of maxiIlary first molars International Endodontic Journal 1992; 25, 304-306

6. Peeters H, Suardita K, Setijanto D. Prevalence of a second canal in the mesiobuccal root of permanent maxillary first molars from an Indonesian population. Journal of Oral Science 2011; Vol. 53, No. 4, 489-494.

7. Das S, Warhadpande M, Redii S, Jibhkate N, Sabir H. Frequency of second mesiobuccal canal in permanent maxillary first molars using the operating microscope and selective dentin removal: A clinical study. Contemporary Clinical Dentistry. Jan-Mar 2015; Vol 6. 74-78.

8. Karaman G. Onay E. Ungor M. Colak M. Evaluating the potential key factors in assessing the morphology of mesiobuccal canal in maxillary first and second molars. Australian Endodontic Journal 2011; 37: 134-140.

9. Hosova N, Yoshida T, lino F, Arai T, Mishima A, Kobayashi K. Detection of a secondary mesio-buccal canal in maxillary first molar: A comparative study Journal of Conservative Dentistry. Apr-Jun 2012, Vol 15, 127-131.

10. Cleghorn BM, Christie WH, Dong CC. Root and root canal morphology of the human permanent maxillary first molar: a literature review. J Endod 2006; 32:813-21.

11. Buhrley LJ, Barrows MJ, BeGole EA, Wenckus CS. Effect of magnification on locating the MB2 canal in maxillary molars. Journal of Endodontics 2002; 28, 324-7.

12. Alaçam, Tinaz A, Geç Ö, Kayaoglu G. Second mesiobuccal canal detection in maxillary first molars using microscopy and ultrasonics. Australian Endodontic Journal 2008; 34: 106-109

13. Yoshioka T, Kikuchi I, Fukumoto Y, Kobayashi C, Suda. Detection of the second mesiobuccal canal in mesiobuccal roots of maxillary molar teeth ex vivo. International Endodontic Journal 2005: 38, 124-128. 